\title{
Nonlocal FEM Formulation for Vibration Analysis of Nanowires on Elastic Matrix with Different Materials
}

\author{
Büşra Uzun ${ }^{1}$ and Ömer Civalek ${ }^{2, *}$ \\ 1 Civil Engineering Department, Uludağ University, 16059 Bursa, Turkey; uzunbusra34@gmail.com \\ 2 Civil Engineering Department, Akdeniz University, 07070 Antalya, Turkey \\ * Correspondence: civalek@yahoo.com
}

Received: 12 February 2019; Accepted: 4 April 2019; Published: 6 April 2019

\begin{abstract}
In this study, free vibration behaviors of various embedded nanowires made of different materials are investigated by using Eringen's nonlocal elasticity theory. Silicon carbide nanowire (SiCNW), silver nanowire (AgNW), and gold nanowire (AuNW) are modeled as Euler-Bernoulli nanobeams with various boundary conditions such as simply supported (S-S), clamped simply supported (C-S), clamped-clamped (C-C), and clamped-free (C-F). The interactions between nanowires and medium are simulated by the Winkler elastic foundation model. The Galerkin weighted residual method is applied to the governing equations to gain stiffness and mass matrices. The results are given by tables and graphs. The effects of small-scale parameters, boundary conditions, and foundation parameters on frequencies are examined in detail. In addition, the influence of temperature change on the vibrational responses of the nanowires are also pursued as a case study.
\end{abstract}

Keywords: nonlocal elasticity theory; Galerkin weighted residual FEM; silicon carbide nanowire; silver nanowire; gold nanowire

\section{Introduction}

Nanoscale structures/materials have very different characteristics. Nanostructures/nanomaterials have attracted great attention because their extraordinary features, such as high strength, low density, high elasticity modulus, and high hardness [1-4], have become the focus of researchers. The aforementioned unique properties of such structures, materials, and rapid developments in nanotechnology has led to use of these structural elements in designing micro- and nanoelectro mechanical systems (MEMS and NEMS) such as resonators, atomic force microscopes, switches, actuators, and sensors.

Some experimental studies have revealed the deformation behaviors of micro-/nanosized structures $[5,6]$. However, experiments are very difficult and quite expensive on these scales because high precision test devices are needed. On the other hand, atomistic modeling such as molecular dynamic simulations is computationally expensive and requires a long period of time. Consequently, this option is limited to structures that have only a few atoms [7].

To understand and accurately interpret the mechanical properties and behaviors of nanoscale structures, use of models based on continuum mechanics may be a better alternative than experiments and atomistic modelling. Unfortunately, classical continuum theories are not sufficient to predict and estimate size dependency because they lack internal/additional material length scale parameters. In ultrasmall scales (micrometer dimension, nanometer dimension) interactions between atoms and molecules have increasing importance and cannot be neglected. Therefore, the solution to classical continuum theories, which does not take into account size effects, does not give accurate results. In order to obtain more accurate results, higher-order continuum theories such as couple stress theory [8-10], modified couple stress theory [11], strain gradient theory [12], modified strain gradient theory [13], and nonlocal elasticity theory [14] have been developed and contain various length scale 
parameters. These theories were used by many researchers for various analyses such as buckling [15], bending [16,17], free vibration [18,19], forced vibration [20], and nonlinear vibration [21].

Rahmanian et al. [22] presented free vibrations of single-walled carbon nanotubes (SWCNT) on a Winkler elastic foundation via nonlocal elasticity theory. In this study, SWCNT was modeled as both beam and shell structures. Demir and Civalek [23] reported thermal vibration formulation of a nonlocal Euler-Bernoulli beam embedded in an elastic matrix. Finite element formulation for Eringen's nonlocal elasticity theory was employed via Hermitian cubic shape functions. Thermal vibrational behaviors of silicon carbide nanowire on an elastic matrix were investigated for simply supported (S-S) and clamped-clamped (C-C) boundary conditions. Finite element formulations of nonlocal elastic Euler-Bernoulli and Timoshenko beam theories were achieved by Pradhan [24]. Vibration, buckling, and bending analyses of carbon nanotubes with four different boundary conditions were performed by the Galerkin finite element technique. Rajasekaran and Bakhshi Khaniki [25] reported static deformation, stability, and free vibration responses of small-scale beams. A finite element model of axial, functionally graded, nonuniform small-scale beams was investigated by using nonlocal strain gradient theory. Eltaher et al. [26] pursued free vibration analysis of functionally graded Euler-Bernoulli nanobeams by using Eringen's nonlocal elasticity theory. Finite element results were given for a dynamic analysis of the nanobeam. Nejad and Hadi [27] studied bending analysis of non-homogeneous nanobeams. Eringen's nonlocal elasticity theory was utilized in Euler-Bernoulli nanobeams made of bi-directional, functionally graded material. Murmu and Pradhan [28] studied the thermo-mechanical vibration response of embedded carbon nanotubes surrounded by an elastic matrix based on nonlocal elasticity theory. Reddy [29] developed nonlocal beam models based on four different beam theories. Static bending, free vibration, and buckling analyses of nanobeams are performed in this study. Tornabene et al. [30] presented a multiscale approach for three-phase carbon nanotube (CNT)/polymer/fiber-laminated nanocomposite structures. Detailed formulations can be found in the literature [31-35] about CNT-reinforcement or the finite element method (FEM). More recently, Uzun et al. [36] investigated the free vibration responses of carbon nanotubes and boron nitride nanotubes based on nonlocal elasticity theory. Nonlocal natural frequencies are obtained for various cross-section geometries.

In the present study, free vibration analysis of three kinds of nanowires resting on a Winkler elastic foundation with various boundary conditions are performed. Simply supported (S-S), clamped simply supported (C-S), clamped-clamped (C-C), and clamped-free (C-F) boundary conditions are selected. Silicon carbide nanowire ( $\mathrm{SiCNW}$ ), silver nanowire (AgNW), and gold nanowire (AuNW) are modeled as nonlocal Euler-Bernoulli beams, and their vibration behaviors are investigated using the finite element method (FEM). A Galerkin weighted residual method is utilized to govern equations and matrices, and the Winkler foundation parameter and small-scale parameter are gained. Effects of boundary conditions, temperature rise, and small-scale and Winkler foundation parameters of frequency values are investigated and compared for three kinds of nanowires.

\section{Euler-Bernoulli Nanobeam Resting on a Winkler Elastic Foundation}

The nonlocal stress tensor $\sigma_{i j}$ at point $x$ is expressed as follows [14]:

$$
\begin{gathered}
\sigma_{i j, j}=0, \\
\left.\sigma_{i j}(x)=\int_{\Omega} K\left(\left|x^{\prime}-x\right|\right), \tau\right) C_{i j k l} \varepsilon_{k l} d \Omega\left(x^{\prime}\right),
\end{gathered}
$$

where $K\left(\left|x^{\prime}-x\right|, \tau\right)$ is the Kernel function, $\left|x^{\prime}-x\right|$ is the distance in the Euclidean form, $\tau=e_{0} a / l$ is a material constant that depends upon the internal characteristic lengths $(a)$ and external characteristic length $(l)$, and $e_{0}$ is a material constant that is determined experimentally. $C_{i j k l}$ and $\varepsilon_{k l}$ represent the fourth-order elasticity and the strain tensors, respectively, and $\Omega$ is the region occupied by the body. 
The nonlocal constitutive formulation is [28]:

$$
\left[1-\tau^{2} l^{2} \nabla^{2}\right] \sigma_{i j}=C_{i j k l} \varepsilon_{k l} .
$$

$x, y, z$ depict length, width, and height of the beam, respectively and $u_{1}, u_{2}, u_{3}$ are the displacements in the $x, y, z$ directions. The displacements for a Bernoulli-Euler beam can be written as below [17]:

$$
u_{1}(x, z, t)=-z \frac{\partial w(x, t)}{\partial x}, u_{2}(x, z, t)=0, u_{3}(x, z, t)=w(x, t) .
$$

$\varepsilon_{i j}$ is the strain tensor, expressed as:

$$
\varepsilon_{i j}=0.5\left(\partial u_{i, j}+\partial u_{j, i}\right)
$$

From Equation (5) we find the strains of the Euler-Bernoulli beam as follows:

$$
\varepsilon_{x x}=-z \frac{\partial^{2} w(x, t)}{\partial x^{2}}, \varepsilon_{x y}=\varepsilon_{y x}=\varepsilon_{x z}=\varepsilon_{z x}=\varepsilon_{y y}=\varepsilon_{y z}=\varepsilon_{z y}=\varepsilon_{z z}=0 .
$$

Stress $\sigma$ for the linear elastic materials is expressed as follows:

$$
\sigma=E \varepsilon,
$$

where $E$ is the elastic modulus of the material. If $\varepsilon_{x x}$, the only nonzero component of strain, is written in Equation (7), $\sigma_{x x}$ is obtained as:

$$
\sigma_{x x}=-E z \frac{\partial^{2} w(x, t)}{\partial x^{2}}
$$

Moment $(M)$ and the moment of inertia $(I)$ are given by:

$$
M=\int_{A} z \sigma_{x x} d A, I=\int_{A} z^{2} d A,
$$

where $A$ represents the cross-section area.

For the transverse vibration of an Euler-Bernoulli beam (shown in Figure 1) resting on a Winkler elastic foundation, the equilibrium conditions are:

$$
\begin{gathered}
\frac{\partial V(x, t)}{\partial x}=-q(x, t)+\rho A \frac{\partial^{2} w(x, t)}{\partial x^{2}}+k_{w} w(x, t), \\
V(x, t)=\frac{\partial M(x, t)}{\partial x}, \\
\frac{\partial^{2} M(x, t)}{\partial x^{2}}=-q(x, t)+\rho A \frac{\partial^{2} w(x, t)}{\partial x^{2}}+k_{w} w(x, t),
\end{gathered}
$$

where $\rho, q(x, t)$, and $k_{w}$ are the mass density, distributed load, and Winkler foundation parameter, respectively.

The nonlocal constitutive relations can be simplified in the following form for a one-dimensional case [14,29]:

$$
\sigma_{x x}-\left(e_{0} a\right)^{2} \frac{\partial^{2} \sigma_{x x}}{\partial x^{2}}=E \varepsilon_{x x}
$$

By multiplying $z$ on both sides of Equation (13) and integrating the cross-sectional area of the beam, we obtain:

$$
\int_{A} z \sigma d A-\left(e_{0} a\right)^{2} \int_{A} z \frac{\partial^{2} \sigma}{\partial x^{2}} d A=\int_{A} z E \varepsilon d A=0 .
$$


Substituting Equations (6) and (9) into (14), we get:

$$
M(x, t)-\left(e_{0} a\right)^{2} \frac{\partial^{2} M(x, t)}{\partial x^{2}}=-E I \frac{\partial^{2} w(x, t)}{\partial x^{2}} .
$$

By differentiating Equation (15) twice with respect to variable $x$ and substituting Equation (12) into Equation (15), we obtain the governing equation for the vibration of an Euler-Bernoulli nanobeam resting on a Winkler elastic foundation, as below:

$$
E I \frac{\partial^{4} w(x, t)}{\partial x^{4}}+\rho A \frac{\partial w^{2}(x, t)}{\partial t^{2}}+k_{w} w-\left(e_{0} a\right)^{2} \frac{\partial^{2}}{\partial x^{2}}\left[\rho A \frac{\partial^{2} w(x, t)}{\partial t^{2}}+k_{w} w\right]=0 .
$$

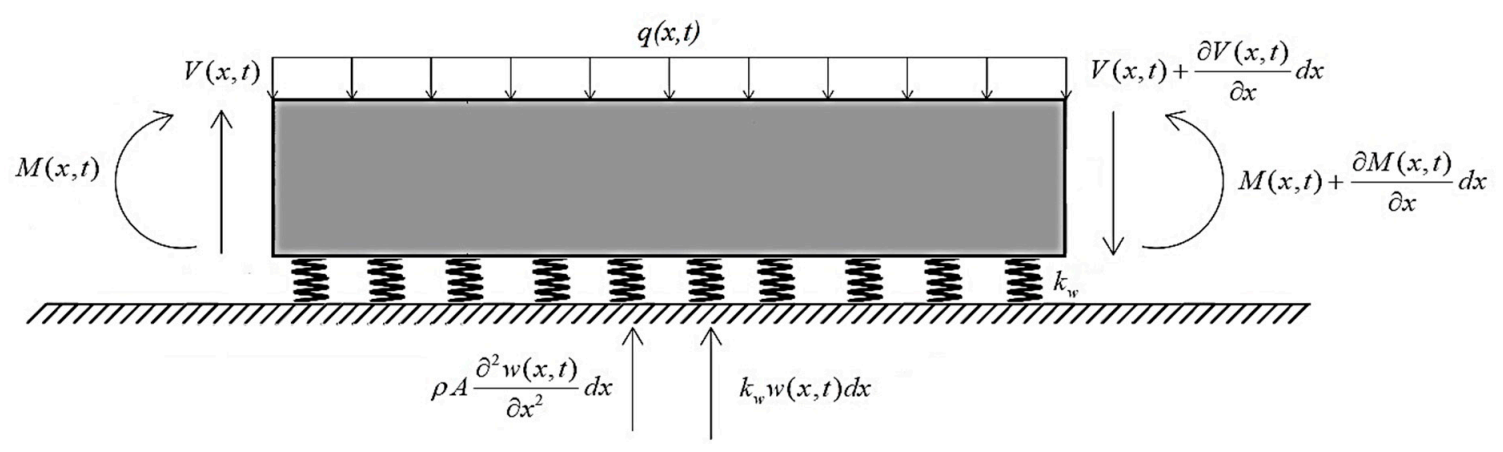

Figure 1. Euler-Bernoulli beam resting on a Winkler foundation.

\section{Solution to the Vibration Problem}

\subsection{Galerkin Weighted Residual Method}

The shape function for beam $\varphi$ is as follows [37,38]:

$$
\varphi=\left\{\begin{array}{l}
\varphi_{1} \\
\varphi_{2} \\
\varphi_{3} \\
\varphi_{4}
\end{array}\right\}=\left\{\begin{array}{c}
1-3 \xi^{2}+2 \xi^{3} \\
L\left(\xi-2 \xi^{2}+\xi^{3}\right) \\
3 \xi^{2}-2 \xi^{3} \\
L\left(-\xi^{2}+\xi^{3}\right)
\end{array}\right\},
$$

where $\xi=x / L$ is a non-dimensional local coordinate. In order to obtain the weak form of the governing equation of an Euler-Bernoulli nanobeam resting on a Winkler elastic foundation, the residue can be expressed as:

$$
\left[E I \frac{\partial^{4} w(x, t)}{\partial x^{4}}+\rho A \frac{\partial w^{2}(x, t)}{\partial t^{2}}+k_{w} w-\left(e_{0} a\right)^{2} \frac{\partial^{2}}{\partial x^{2}}\left(\rho A \frac{\partial^{2} w(x, t)}{\partial t^{2}}+k_{w} w\right)\right]=\text { residue. }
$$

To determine the weighted residue, Equation (18) is multiplied by a weighting function $\varphi$. When the weighted residual is integrated over the length:

$$
\int_{0}^{L}\left[\varphi E I \frac{\partial^{4} w(x, t)}{\partial x^{4}}+\varphi \rho A \frac{\partial w^{2}(x, t)}{\partial t^{2}}+\varphi k_{w} w-\varphi\left(e_{0} a\right)^{2} \frac{\partial^{2}}{\partial x^{2}}\left(\rho A \frac{\partial^{2} w(x, t)}{\partial t^{2}}+k_{w} w\right)\right] d x=0
$$

Equation (19) is integrated by parts. According to the chain rule, in the general form:

$$
\int_{0}^{L}\left[E I \frac{\partial^{2} \varphi}{\partial x^{2}} \frac{\partial^{2} \varphi^{T}}{\partial x^{2}}+\rho A \varphi \varphi^{T} \ddot{w}+k_{w} \varphi \varphi^{T}-\left(e_{0} a\right)^{2} \rho A \frac{\partial \varphi}{\partial x} \frac{\partial \varphi^{T}}{\partial x} \ddot{w}-\left(e_{0} a\right)^{2} k_{w} \frac{\partial \varphi}{\partial x} \frac{\partial \varphi^{T}}{\partial x}\right] d x=0 .
$$


By using the shape function in Equation (17) and the non-dimensional local coordinate, bending stiffness matrix $K^{b}$, Winkler foundation stiffness matrix $K^{w}$, and the mass matrix $M$, the following are obtained:

$$
\begin{aligned}
& K^{b}=E I \int_{0}^{L}\left\{\begin{array}{c}
\varphi_{1}^{\prime \prime} \\
\varphi_{2}^{\prime \prime} \\
\varphi_{3}^{\prime \prime} \\
\varphi_{4}^{\prime \prime}
\end{array}\right\}\left\{\begin{array}{llll}
\varphi_{1}^{\prime \prime} & \varphi_{2}^{\prime \prime} & \varphi_{3}^{\prime \prime} & \varphi_{4}^{\prime \prime}
\end{array}\right\} d x \\
& K^{b}=\frac{E I}{L^{3}}\left[\begin{array}{cccc}
12 & 6 L & -12 & 6 L \\
6 L & 4 L^{2} & -6 L & 2 L^{2} \\
-12 & -6 L & 12 & -6 L \\
6 L & 2 L^{2} & -6 L & 4 L^{2}
\end{array}\right] \\
& K^{w 1}=k_{w} \int_{0}^{L}\left\{\begin{array}{c}
\varphi_{1} \\
\varphi_{2} \\
\varphi_{3} \\
\varphi_{4}
\end{array}\right\}\left\{\begin{array}{llll}
\varphi_{1} & \varphi_{2} & \varphi_{3} & \varphi_{4}
\end{array}\right\} d x \\
& K^{w 1}=\frac{k_{w}}{420}\left[\begin{array}{cccc}
156 L & 22 L^{2} & 54 L & -13 L^{2} \\
22 L^{2} & 4 L^{3} & 13 L^{2} & -3 L^{3} \\
54 L & 13 L^{2} & 156 L & -22 L^{2} \\
-13 L^{2} & -3 L^{3} & -22 L^{2} & 4 L^{3}
\end{array}\right] \\
& K^{w 2}=\left(e_{0} a\right)^{2} k_{w} \int_{0}^{L}\left\{\begin{array}{c}
\varphi_{1}^{\prime} \\
\varphi_{2}^{\prime} \\
\varphi_{3}^{\prime} \\
\varphi_{4}^{\prime}
\end{array}\right\}\left\{\begin{array}{llll}
\varphi_{1}^{\prime} & \varphi_{2}^{\prime} & \varphi_{3}^{\prime} & \varphi_{4}^{\prime}
\end{array}\right\} d x ; \\
& K^{w 2}=\frac{\left(e_{0} a\right)^{2} k_{w}}{30 L}\left[\begin{array}{cccc}
36 & 3 L & -36 & 3 L \\
3 L & 4 L^{2} & -3 L & -L^{2} \\
-36 & -3 L & 36 & -3 L \\
3 L & -L^{2} & -3 L & 4 L^{2}
\end{array}\right] \\
& M^{1}=\rho A \int_{0}^{L}\left\{\begin{array}{c}
\varphi_{1} \\
\varphi_{2} \\
\varphi_{3} \\
\varphi_{4}
\end{array}\right\}\left\{\begin{array}{cccc}
\varphi_{1} & \varphi_{2} & \varphi_{3} & \varphi_{4}
\end{array}\right\} d x \\
& M^{1}=\frac{\rho A}{420}\left[\begin{array}{cccc}
156 L & 22 L & 54 L & -13 L^{2} \\
22 L^{2} & 4 L^{3} & 13 L^{2} & -3 L^{3} \\
54 L & 13 L^{2} & 156 L & -22 L^{2} \\
-13 L^{2} & -3 L^{3} & -22 L^{2} & 4 L^{3}
\end{array}\right] \text {; } \\
& M^{2}=\left(e_{0} a\right)^{2} \rho A \int_{0}^{L}\left\{\begin{array}{c}
\varphi_{1}^{\prime} \\
\varphi_{2}^{\prime} \\
\varphi_{3}^{\prime} \\
\varphi_{4}^{\prime}
\end{array}\right\}\left\{\begin{array}{llll}
\prime & \varphi_{2}^{\prime} & \varphi_{3}^{\prime} & \varphi_{4}^{\prime}
\end{array}\right\} d x \\
& M^{2}=\frac{\left(e_{0} a\right)^{2} \rho A}{30 L}\left[\begin{array}{cccc}
36 & 3 L & -36 & 3 L \\
3 L & 4 L^{2} & -3 L & -L^{2} \\
-36 & -3 L & 36 & -3 L \\
3 L & -L^{2} & -3 L & 4 L^{2}
\end{array}\right] \text {. }
\end{aligned}
$$


The vibration of the Euler-Bernoulli beam is found as follows:

$$
\left|K-\omega^{2} M\right|=0,
$$

where $K=K^{\mathrm{b}}+K^{w 1}+K^{w 2}, M=M^{1}+M^{2}$, and $\omega$ is frequency.

\subsection{Thermal Effect on the Vibrational Response of Embedded Nanowires}

Here, the effect of temperature change on the natural frequencies of embedded nanowires in a thermal environment is investigated. Equation (16) can be rewritten in the presence of thermal loading as:

$$
\left(E I-N^{T}\left(e_{0} a\right)^{2}\right) \frac{\partial^{4} w}{\partial x^{4}}+k_{w} w+\left(N^{T}-k_{w}\left(e_{0} a\right)^{2}\right) \frac{\partial^{2} w}{\partial x^{2}}+\rho A \frac{\partial^{2} w}{\partial t^{2}}-\rho A\left(e_{0} a\right)^{2} \frac{\partial^{4} w}{\partial x^{2} \partial t^{2}}=0,
$$

where the additional term $N^{T}$ is the axial load resulting from the temperature change and can be defined as [28]:

$$
N^{T}=\frac{E A}{1-2 v} \alpha \Delta T
$$

in which $\alpha$ is the thermal expansion coefficient in the axial direction, $\Delta T$ is the temperature change, and $v$ is Poisson's ratio. It is notable that only an axial load resulting from temperature change exists for the nanowires in this study [28].

The following Navier's solution procedure is applied to achieve simply supported (S-S) nanowires as an illustrative example:

$$
w(x, t)=\sum_{n=1}^{\infty} W_{n} \sin \beta e^{i \omega_{n} t},
$$

where $n$ is the mode number, $W_{n}$ is the unknown Fourier coefficient, and $\beta=\frac{n \pi x}{L}$. Using Equation (29) in Equation (27) yields the following relation for small-scale-dependent natural frequencies of embedded S-S nanowires that includes the thermal effect:

$$
\omega_{n}=\sqrt{\frac{\beta^{4}\left(E I-N^{T}\left(e_{0} a\right)^{2}\right)+k_{w}-\beta^{2}\left(N^{T}-k_{w}\left(e_{0} a\right)^{2}\right)}{\rho A\left(1+\beta^{2}\left(e_{0} a\right)^{2}\right)}} .
$$

\section{Results and Discussion}

In this section, frequency values of nanowires were obtained with various non-dimensional small-scale parameters $\left(e_{0} a / L\right)$, different non-dimensional Winkler foundation parameters $\left(K_{W}\right)$, different boundary conditions, and different number of elements $(N)$. The material properties for the three nanowires are listed in Table 1 . The results obtained were shown in tables and graphs. The dimensionless Winkler parameter used for the results is expressed as the formula below:

$$
K_{W}=\frac{k_{w} L^{4}}{E I}
$$

Table 1. Material properties of the nanowires.

\begin{tabular}{cccc}
\hline Material Properties & SiCNW & AuNW & AgNW \\
\hline$E(\mathrm{GPa})$ & 524.8 & 79 & 82.7 \\
$v$ & 0.14 & 0.42 & 0.37 \\
$\rho\left(\mathrm{kg} / \mathrm{m}^{3}\right)$ & 3100 & 19,320 & 10,490 \\
$\alpha(1 /)$ & $3.7 \times 10^{-6}$ & $14.2 \times 10^{-6}$ & $19.68 \times 10^{-6}$ \\
\hline
\end{tabular}


Table 2 presents the natural frequencies of nanowires with C-C, C-S, S-S, and C-F boundary conditions. Finite element solutions for the three nanowires were compared with each other. It was clearly observed from the table that the highest frequency value occurred for both $\mathrm{SiCNW}$ and the C-C boundary condition, while the lowest value was seen in both AuNW and C-F boundary conditions.

Table 2. The first three natural frequencies $(\mathrm{GHz})$ of isolated nanowires for four different boundary conditions $\left(K_{W}=0, e_{0} a / L=0.1\right)$.

\begin{tabular}{ccccc}
\hline \multirow{2}{*}{ Mode Number } & \multicolumn{4}{c}{ SiCNW } \\
\cline { 2 - 5 } & C-C & C-S & S-S & C-F \\
\hline $\mathbf{1}$ & 21.8286 & 15.0969 & 9.7369 & 3.5540 \\
$\mathbf{2}$ & 52.7211 & 43.2195 & 34.5672 & 19.7887 \\
$\mathbf{3}$ & 88.6384 & 77.4034 & 66.8450 & 48.0785 \\
\hline \multirow{4}{*}{ Mode Number } & \multicolumn{5}{c}{ AuNW } \\
\cline { 2 - 5 } & C-C & C-S & S-S & C-F \\
\hline $\mathbf{1}$ & 3.3525 & 2.3186 & 1.4954 & 0.5458 \\
$\mathbf{2}$ & 8.0970 & 6.6378 & 5.3089 & 3.0392 \\
$\mathbf{3}$ & 13.6133 & 11.8878 & 10.2662 & 7.3840 \\
\hline \multirow{3}{*}{ Mode Number } & \multicolumn{5}{c}{ AgNW } \\
\cline { 2 - 5 } & C-C & C-S & S-S & C-F \\
\hline $\mathbf{1}$ & 4.6550 & 3.2195 & 2.0764 & 0.7579 \\
$\mathbf{2}$ & 11.2430 & 9.2167 & 7.3716 & 4.2200 \\
$\mathbf{3}$ & 18.9025 & 16.5066 & 14.2550 & 10.2529 \\
\hline
\end{tabular}

Tables 3-6 show natural frequencies of embedded simply supported nanowires for $K_{W}=1$, $K_{W}=10, K_{W}=100$, and $K_{W}=1000$, respectively, with different $e_{0} a / L$ values. It was found from these tables that frequency values increased as $K_{W}$ values increased, but frequencies decreased by increasing $e_{0} a / L$. Moreover, it was evident that small-scale effects became more considerable for higher modes.

Table 3. The first three natural frequencies $(\mathrm{GHz})$ of embedded nanowires corresponding to various values of $e_{0} a / L\left(K_{W}=1\right)$.

\begin{tabular}{|c|c|c|c|c|}
\hline \multirow{3}{*}{ Mode Number } & \multicolumn{4}{|c|}{ SiCNW } \\
\hline & \multicolumn{4}{|c|}{$e_{0} a / L$} \\
\hline & 0.0 & 0.1 & 0.2 & 0.3 \\
\hline 1 & 10.2583 & 9.7916 & 8.7034 & 7.4989 \\
\hline 2 & 40.8373 & 34.5827 & 25.4413 & 19.1602 \\
\hline \multirow[t]{2}{*}{3} & 91.8603 & 66.8530 & 43.0600 & 30.6452 \\
\hline & \multicolumn{4}{|c|}{ AuNW } \\
\hline \multirow[t]{2}{*}{ Mode Number } & \multicolumn{4}{|c|}{$e_{0} a / L$} \\
\hline & 0.0 & 0.1 & 0.2 & 0.3 \\
\hline 1 & 1.5755 & 1.5038 & 1.3367 & 1.1517 \\
\hline 2 & 6.2719 & 5.3113 & 3.9073 & 2.9427 \\
\hline \multirow[t]{2}{*}{3} & 14.1081 & 10.2675 & 6.6133 & 4.7066 \\
\hline & \multicolumn{4}{|c|}{ AgNW } \\
\hline \multirow[t]{2}{*}{ Mode Number } & \multicolumn{4}{|c|}{$e_{0} a / L$} \\
\hline & 0.0 & 0.1 & 0.2 & 0.3 \\
\hline 1 & 2.1876 & 2.0881 & 1.8560 & 1.5992 \\
\hline 2 & 8.7087 & 7.3749 & 5.4255 & 4.0860 \\
\hline 3 & 19.5896 & 14.2567 & 9.1827 & 6.5352 \\
\hline
\end{tabular}


Table 4. The first three natural frequencies $(\mathrm{GHz})$ of embedded nanowires for various values of $e_{0} a / L$ $\left(K_{W}=10\right)$.

\begin{tabular}{|c|c|c|c|c|}
\hline \multirow{3}{*}{ Mode Number } & \multicolumn{4}{|c|}{ SiCNW } \\
\hline & \multicolumn{4}{|c|}{$e_{0} a / L$} \\
\hline & 0.0 & 0.1 & 0.2 & 0.3 \\
\hline 1 & 10.7171 & 10.2713 & 9.2398 & 8.1152 \\
\hline 2 & 40.9550 & 34.7215 & 25.6298 & 19.4097 \\
\hline 3 & 91.9126 & 66.9249 & 43.1716 & 30.8018 \\
\hline \multirow{3}{*}{ Mode Number } & \multicolumn{4}{|c|}{ AuNW } \\
\hline & \multicolumn{4}{|c|}{$e_{0} a / L$} \\
\hline & 0.0 & 0.1 & 0.2 & 0.3 \\
\hline 1 & 1.6460 & 1.5775 & 1.4191 & 1.2464 \\
\hline 2 & 6.2900 & 5.3326 & 3.9363 & 2.9810 \\
\hline 3 & 14.1162 & 10.2785 & 6.6304 & 4.7306 \\
\hline \multirow{3}{*}{ Mode Number } & \multicolumn{4}{|c|}{ AgNW } \\
\hline & \multicolumn{4}{|c|}{$e_{0} a / L$} \\
\hline & 0.0 & 0.1 & 0.2 & 0.3 \\
\hline 1 & 2.2855 & 2.1904 & 1.9704 & 1.7306 \\
\hline 2 & 8.7338 & 7.4045 & 5.4656 & 4.1392 \\
\hline 3 & 19.6007 & 14.2720 & 9.2065 & 6.5686 \\
\hline
\end{tabular}

Table 5. The first three natural frequencies $(\mathrm{GHz})$ of embedded nanowires with respect to various values of $e_{0} a / L\left(K_{W}=100\right)$.

\begin{tabular}{|c|c|c|c|c|}
\hline \multirow{3}{*}{ Mode Number } & \multicolumn{4}{|c|}{ SiCNW } \\
\hline & \multicolumn{4}{|c|}{$e_{0} a / L$} \\
\hline & 0.0 & 0.1 & 0.2 & 0.3 \\
\hline 1 & 14.5292 & 14.2035 & 13.4764 & 12.7318 \\
\hline 2 & 42.1135 & 36.0808 & 27.4431 & 21.7480 \\
\hline \multirow[t]{2}{*}{3} & 92.4347 & 67.6401 & 44.2722 & 32.3263 \\
\hline & \multicolumn{4}{|c|}{ AuNW } \\
\hline \multirow[t]{2}{*}{ Mode Number } & \multicolumn{4}{|c|}{$e_{0} a / L$} \\
\hline & 0.0 & 0.1 & 0.2 & 0.3 \\
\hline 1 & 2.2314 & 2.1814 & 2.0697 & 1.9554 \\
\hline 2 & 6.4679 & 5.5414 & 4.2148 & 3.3401 \\
\hline \multirow[t]{2}{*}{3} & 14.1964 & 10.3883 & 6.7994 & 4.9648 \\
\hline & \multicolumn{4}{|c|}{ AgNW } \\
\hline \multirow[t]{2}{*}{ Mode Number } & \multicolumn{4}{|c|}{$e_{0} a / L$} \\
\hline & 0.0 & 0.1 & 0.2 & 0.3 \\
\hline 1 & 3.0984 & 3.0290 & 2.8739 & 2.7151 \\
\hline 2 & 8.9809 & 7.6944 & 5.8524 & 4.6379 \\
\hline 3 & 19.7121 & 14.4245 & 9.4412 & 6.8937 \\
\hline
\end{tabular}


Table 6. The first three natural frequencies $(\mathrm{GHz})$ of embedded nanowires against various values of $e_{0} a / L\left(K_{W}=1000\right)$.

\begin{tabular}{|c|c|c|c|c|}
\hline \multirow{3}{*}{ Mode Number } & \multicolumn{4}{|c|}{ SiCNW } \\
\hline & \multicolumn{4}{|c|}{$e_{0} a / L$} \\
\hline & 0.0 & 0.1 & 0.2 & 0.3 \\
\hline 1 & 34.2564 & 34.1196 & 33.8234 & 33.5336 \\
\hline 2 & 52.3064 & 47.5839 & 41.4190 & 37.8864 \\
\hline \multirow[t]{2}{*}{3} & 97.5017 & 74.4150 & 54.0595 & 44.8040 \\
\hline & \multicolumn{4}{|c|}{ AuNW } \\
\hline \multirow[t]{2}{*}{ Mode Number } & \multicolumn{4}{|c|}{$e_{0} a / L$} \\
\hline & 0.0 & 0.1 & 0.2 & 0.3 \\
\hline 1 & 5.2612 & 5.2402 & 5.1947 & 5.1502 \\
\hline 2 & 8.0333 & 7.3081 & 6.3612 & 5.8187 \\
\hline \multirow[t]{2}{*}{3} & 14.9746 & 11.4288 & 8.3026 & 6.8811 \\
\hline & \multicolumn{4}{|c|}{ AgNW } \\
\hline \multirow[t]{2}{*}{ Mode Number } & \multicolumn{4}{|c|}{$e_{0} a / L$} \\
\hline & 0.0 & 0.1 & 0.2 & 0.3 \\
\hline 1 & 7.3053 & 7.2761 & 7.2130 & 7.1512 \\
\hline 2 & 11.1545 & 10.1475 & 8.8328 & 8.0794 \\
\hline 3 & 20.7926 & 15.8693 & 11.5284 & 9.5546 \\
\hline
\end{tabular}

In Table 7, the frequency values of C-C nanowires are given by analytical and finite element solutions for $e_{0} a / L=0.2$. For the finite element solution, as the element number increased, the results approached the real value.

Effects of both temperature rise and the Winkler parameter on the first three natural frequencies of nanowires are revealed in Table 8. It is apparent from the table that an increase temperature rise led to a decrease in frequency, contrary to the Winkler parameter. Also, it can be emphasized that the frequencies of AuNW were more affected than the other nanowires because of their related material properties, given in Table 1. Moreover, it can be observed that the influence of temperature rise was more prominent for lower modes and smaller Winkler parameters.

Table 7. Convergence of the present results with the analytical results of different element numbers.

\begin{tabular}{|c|c|c|c|c|c|c|c|c|}
\hline \multirow{2}{*}{ Mode Number } & \multicolumn{8}{|c|}{ SiCNW } \\
\hline & $N=4$ & $N=5$ & $N=6$ & $N=7$ & $N=8$ & $N=9$ & $N=10$ & Analytical \\
\hline 1 & 18.9418 & 18.9252 & 18.9189 & 18.9162 & 18.9148 & 18.9141 & 18.9137 & 18.9129 \\
\hline 2 & 38.0339 & 37.8398 & 37.7549 & 37.7155 & 37.6955 & 37.6845 & 37.6781 & 37.6656 \\
\hline 3 & 56.8236 & 57.2104 & 56.8478 & 56.6515 & 56.5467 & 56.4878 & 56.4529 & 56.3826 \\
\hline \multirow{2}{*}{ Mode Number } & \multicolumn{8}{|c|}{ AuNW } \\
\hline & $N=4$ & $N=5$ & $N=6$ & $N=7$ & $N=8$ & $N=9$ & $N=10$ & Analytical \\
\hline 1 & 2.9091 & 2.9066 & 2.9056 & 2.9052 & 2.9050 & 2.9049 & 2.9048 & 2.9047 \\
\hline 2 & 5.8413 & 5.8115 & 5.7985 & 5.7924 & 5.7894 & 5.7877 & 5.7867 & 5.7848 \\
\hline 3 & 8.7271 & 8.7865 & 8.7308 & 8.7007 & 8.6846 & 8.6755 & 8.6702 & 8.6594 \\
\hline \multirow{2}{*}{ Mode Number } & \multicolumn{8}{|c|}{ AgNW } \\
\hline & $N=4$ & $N=5$ & $N=6$ & $N=7$ & $N=8$ & $N=9$ & $N=10$ & Analytical \\
\hline 1 & 4.0394 & 4.0359 & 4.0345 & 4.0340 & 4.0337 & 4.0335 & 4.0334 & 4.0332 \\
\hline 2 & 8.1109 & 8.0695 & 8.0514 & 8.0430 & 8.0387 & 8.0364 & 8.0350 & 8.0323 \\
\hline 3 & 12.1179 & 12.2003 & 12.1230 & 12.0811 & 12.059 & 12.0462 & 12.0388 & 12.0238 \\
\hline
\end{tabular}


Table 8. The first three small-scale-dependent natural frequencies $(\mathrm{GHz})$ of embedded nanowires for different Winkler parameters and temperature changes $\left(e_{0} a / L=0.1, \mathrm{~d}=1 \mathrm{~nm}\right.$, and $\left.L=20 \mathrm{~d}\right)$.

\begin{tabular}{|c|c|c|c|c|c|c|}
\hline \multirow{3}{*}{ Mode Number } & \multicolumn{6}{|c|}{ SiCNW } \\
\hline & \multicolumn{3}{|c|}{$\Delta T=0$} & \multicolumn{3}{|c|}{$\Delta T=30^{\circ} \mathrm{C}$} \\
\hline & $K w=100$ & $K w=500$ & $K w=1000$ & $K w=100$ & $K w=500$ & $K w=1000$ \\
\hline 1 & 17.7768 & 31.4013 & 42.7034 & 17.3120 & 31.1405 & 42.5119 \\
\hline 2 & 45.1580 & 52.0507 & 59.5551 & 44.4297 & 51.4201 & 59.0047 \\
\hline \multirow[t]{2}{*}{3} & 84.6569 & 88.5259 & 93.1362 & 83.7854 & 87.6928 & 92.3448 \\
\hline & \multicolumn{6}{|c|}{ AuNW } \\
\hline \multirow[t]{2}{*}{ Mode Number } & \multicolumn{3}{|c|}{$\Delta T=0$} & \multicolumn{3}{|c|}{$\Delta T=30^{\circ} \mathrm{C}$} \\
\hline & $K w=100$ & $K w=500$ & $K w=1000$ & $K w=100$ & $K w=500$ & $K w=1000$ \\
\hline 1 & 2.7628 & 4.8802 & 6.6368 & 0.9103 & 4.1246 & 6.1026 \\
\hline 2 & 7.0182 & 8.0895 & 9.2558 & 4.6945 & 6.1824 & 7.6454 \\
\hline \multirow[t]{2}{*}{3} & 13.157 & 13.7583 & 14.4748 & 10.5767 & 11.3159 & 12.177 \\
\hline & \multicolumn{6}{|c|}{ AgNW } \\
\hline \multirow[t]{2}{*}{ Mode Number } & \multicolumn{3}{|c|}{$\Delta T=0$} & \multicolumn{3}{|c|}{$\Delta T=30^{\circ} \mathrm{C}$} \\
\hline & $K w=100$ & $K w=500$ & $K w=1000$ & $K w=100$ & $K w=500$ & $K w=1000$ \\
\hline 1 & 3.8362 & 6.7764 & 9.2153 & 1.8782 & 5.8932 & 8.5868 \\
\hline 2 & 9.7450 & 11.2325 & 12.8519 & 7.0859 & 9.0229 & 10.9734 \\
\hline 3 & 18.2689 & 19.1038 & 20.0987 & 15.2661 & 16.2559 & 17.4143 \\
\hline
\end{tabular}

Effects of nanoscale and foundation parameters on the first five natural frequencies are respectively depicted in Figures 2 and 3. It can be concluded from these figures that size dependency was more pronounced for higher modes, while the natural frequencies in lower modes were more affected from foundation parameters. As stated before, it was clear that the natural frequencies decreased and increased by increasing $e_{0} a / L$ and $K_{W}$, respectively.

Figure 4 displays the variation of fundamental frequencies of the three embedded nanowires with respect to temperature rise for various small-scale parameter values. It can be recognized from the figure that the effect of $e_{0} a / L$ was more prominent for SiCNW than the other ones. On the other hand, the influence of temperature rise was more significant for gold and silver nanowires than the silicon carbide nanowire.

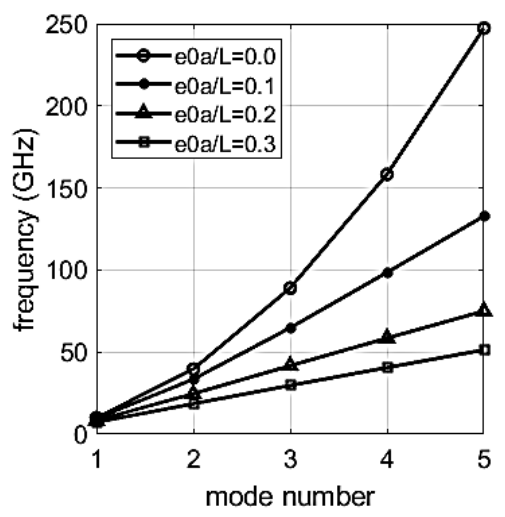

(a) SiCNW

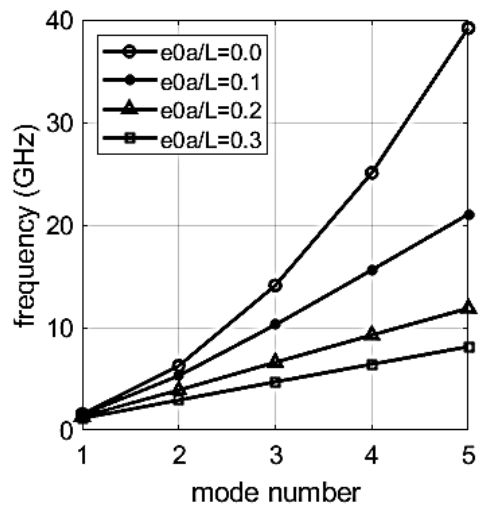

(b) AuNW

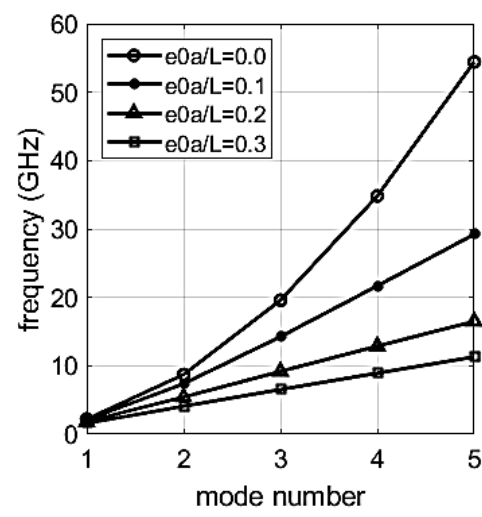

(c) AgNW

Figure 2. Variation of the first five natural frequencies of nanowires with respect to $e_{0} a / L$. 


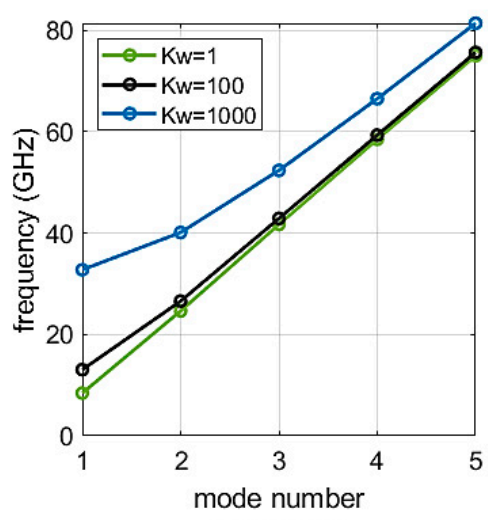

(a) SiCNW

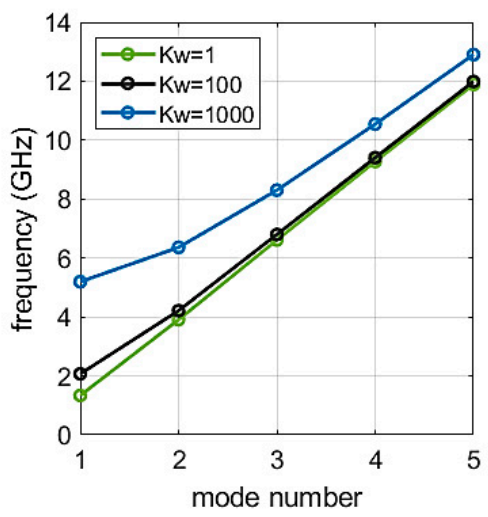

(b) AuNW

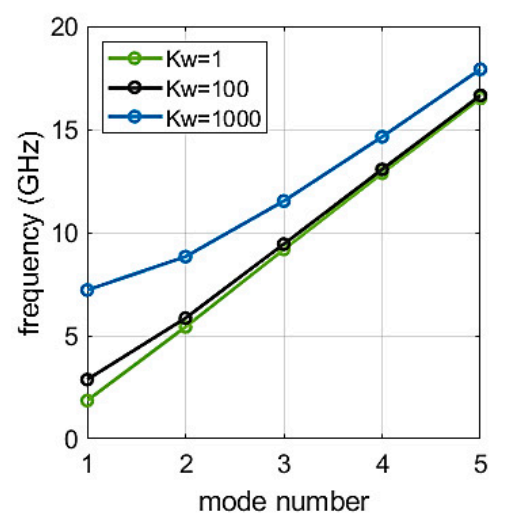

(c) AgNW

Figure 3. Effect of the Winkler parameter on the first five natural frequencies of nanowires.

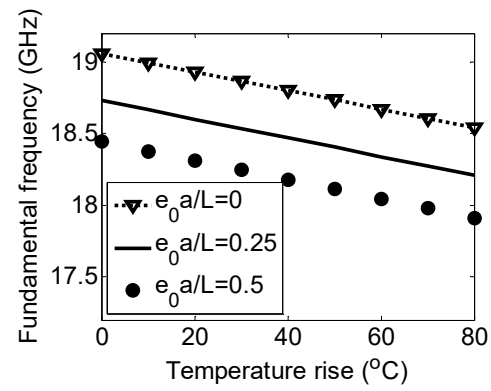

(a) $\mathrm{SiCNW}$

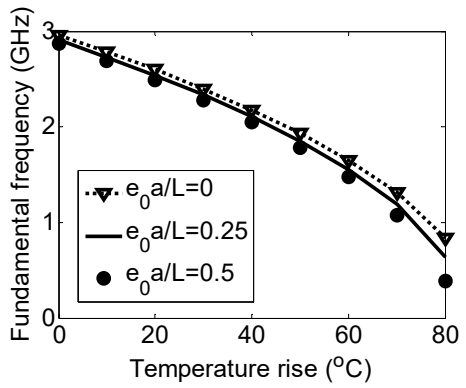

(b) AuNW

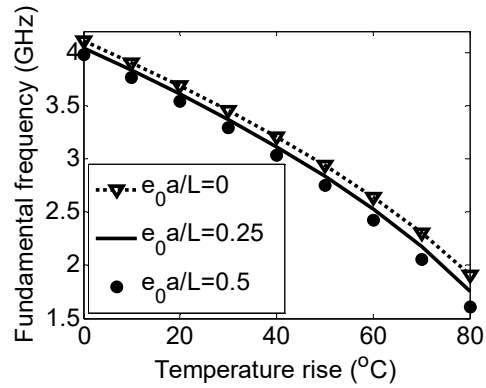

(c) $\mathrm{AgNW}$

Figure 4. Variation of the fundamental frequency for several $e_{0} a / L$ against temperature rise $(L=30 \mathrm{~d}$, $\left.K_{w}=1000\right)$.

\section{Conclusions}

Three types of nanowires, SiCNW, AgNW, and AuNW, are modeled as nonlocal Euler-Bernoulli nanobeams resting on a Winkler elastic foundation. Frequency values of these nanowires are obtained via a finite element solution, and results are given by tables and graphs. The effect of temperature change on the vibrational responses of simply supported nanowires is also examined as a case study. It can be concluded from the results that among all boundary conditions, $\mathrm{C}-\mathrm{C}$ has the highest frequency values and C-F has the lowest ones. Also, it can be emphasized that by increasing the Winkler parameter value, frequency values increase, while by increasing non-dimensional small-scale parameter $\left(e_{0} a / L\right)$, frequency value decreases. When we compare the frequency values of nanowires, SiCNW has the highest frequency values, while AuNW has the lowest frequency values because of its different material properties. Additionally, it is revealed that the effects of temperature change and small-scale parameters on the frequencies of nanowires are both considerable and negligible depending on the values of the involved material properties.

Author Contributions: All authors contributed extensively to the present study. The derivation of the governing equations, obtaining and discussion of the numerical results, and writing the manuscript have been performed by B.U. and Ö.C.

Acknowledgments: This study was supported by The Scientific and Technological Research Council of Turkey (TÜBITAK) with Project no: 117M495. This support is gratefully acknowledged.

Conflicts of Interest: The authors declare no conflict of interest. 


\section{References}

1. Makeev, M.A.; Srivastava, D.; Menon, M. Silicon carbide nanowires under external loads: An atomistic simulation study. Phys. Rev. B 2006, 74, 165303. [CrossRef]

2. Schulz, M.; Shanov, V.; Yin, Z. (Eds.) Nanotube Superfiber Materials: Changing Engineering Design; William Andrew: Norwich, NY, USA, 2013.

3. Numanoğlu, H.M. Nanoyapıların Kiriş ve Çubuk Modellerinin Yerel Olmayan Elastisite Teorisi Kullanılarak Titreşim Analizi; Akdeniz Üniversitesi: Antalya, Turkey, 2017. (In Turkish)

4. Vazinishayan, A.; Yang, S.; Duongthipthewa, A.; Wang, Y. Effects of cross-section on mechanical properties of Au nanowire. AIP Adv. 2016, 6, 025006. [CrossRef]

5. Poole, W.J.; Ashby, M.F.; Fleck, N.A. Micro-hardness of annealed and work-hardened copper polycrystals. Scr. Mater. 1996, 34, 559-564. [CrossRef]

6. Mc Farland, A.W.; Colton, J.S. Role of material microstructure in plate stiffness with relevance to microcantilever sensors. J. Micromech. Microeng. 2005, 15, 1060-1067. [CrossRef]

7. Zhang, C.-L.; Shen, H.-S. Predicting the elastic properties of double- walled carbon nanotubes by molecular dynamics simulation. J. Phys. D Appl. Phys. 2008, 41, 055404. [CrossRef]

8. Toupin, R.A. Elastic materials with couple-stresses. Arch. Ration. Mech. Anal. 1962, 11, 385-414. [CrossRef]

9. Mindlin, R.D.; Tiersten, H.F. Effects of couple-stresses in linear elasticity. Arch. Ration. Mech. Anal. 1962, 11, 415-448. [CrossRef]

10. Koiter, W.T. Couple stresses in the theory of elasticity, I and II. Proc. K. Ned. Akad. Wet. B 1964, 67, 17-44.

11. Yang, F.; Chong, A.C.M.; Lam, D.C.C.; Tong, P. Couple stress based strain gradient theory for elasticity. Int. J. Solids Struct. 2002, 39, 2731-2743. [CrossRef]

12. Mindlin, R.D. Second gradient of strain and surface-tension in linear elasticity. Int. J. Solids Struct. 1965, 1, 417-438. [CrossRef]

13. Lam, D.C.C.; Yang, F.; Chong, A.C.M.; Wang, J.; Tong, P. Experiments and theory in strain gradient elasticity. J. Mech. Phys. Solids 2003, 51, 1477-1508. [CrossRef]

14. Eringen, A.C. On differential equations of nonlocal elasticity and solutions of screw dislocation and surface waves. J. Appl. Phys. 1983, 54, 4703-4710. [CrossRef]

15. Nejad, M.Z.; Hadi, A.; Rastgoo, A. Buckling analysis of arbitrary two-directional functionally graded Euler-Bernoulli nano-beams based on nonlocal elasticity theory. Int. J. Eng. Sci. 2016, 103, 1-10. [CrossRef]

16. Akgöz, B.; Civalek, Ö. Bending analysis of FG microbeams resting on Winkler elastic foundation via strain gradient elasticity. Compos. Struct. 2015, 134, 294-301. [CrossRef]

17. Akgöz, B.; Civalek, Ö. Bending analysis of embedded carbon nanotubes resting on an elastic foundation using strain gradient theory. Acta Astronaut. 2016, 119, 1-12.

18. Togun, N.; Bağdatli, S.M. The vibration of nanobeam resting on elastic foundation using modified couple stress theory. Tehnički Glasnik 2018, 12, 221-225. [CrossRef]

19. Pradhan, S.C.; Phadikar, J.K. Nonlocal elasticity theory for vibration of nanoplates. J. Sound Vib. 2009, 325, 206-223. [CrossRef]

20. Uymaz, B. Forced vibration analysis of functionally graded beams using nonlocal elasticity. Compos. Struct. 2013, 105, 227-239. [CrossRef]

21. Togun, N. Nonlocal beam theory for nonlinear vibrations of a nanobeam resting on elastic foundation. Bound. Value Probl. 2016, 1, 57. [CrossRef]

22. Rahmanian, M.; Torkaman-Asadi, M.A.; Firouz-Abadi, R.D.; Kouchakzadeh, M.A. Free vibrations analysis of carbon nanotubes resting on Winkler foundations based on nonlocal models. Phys. B Condens. Mat. 2016, 484, 83-94. [CrossRef]

23. Demir, Ç.; Civalek, Ö. A new nonlocal FEM via Hermitian cubic shape functions for thermal vibration of nano beams surrounded by an elastic matrix. Compos. Struct. 2017, 168, 872-884. [CrossRef]

24. Pradhan, S.C. Nonlocal finite element analysis and small scale effects of CNTs with Timoshenko beam theory. Finite Elem. Anal. Des. 2012, 50, 8-20. [CrossRef]

25. Rajasekaran, S.; Bakhshi Khaniki, H. Finite element static and dynamic analysis of axially functionally graded nonuniform small-scale beams based on nonlocal strain gradient theory. Mech. Adv. Mat. Struct. 2018, 1-15. [CrossRef] 
26. Eltaher, M.A.; Emam, S.A.; Mahmoud, F.F. Free vibration analysis of functionally graded size-dependent nanobeams. Appl. Math. Comput. 2012, 218, 7406-7420. [CrossRef]

27. Nejad, M.Z.; Hadi, A. Eringen's non-local elasticity theory for bending analysis of bi-directional functionally graded Euler-Bernoulli nano-beams. Int. J. Eng. Sci. 2016, 106, 1-9. [CrossRef]

28. Murmu, T.; Pradhan, S.C. Thermo-mechanical vibration of a single-walled carbon nanotube embedded in an elastic medium based on nonlocal elasticity theory. Comput. Mat. Sci. 2009, 46, 854-859. [CrossRef]

29. Reddy, J.N. Nonlocal theories for bending, buckling and vibration of beams. Int. J. Eng. Sci. 2007, 45, $288-307$. [CrossRef]

30. Tornabene, F.; Bacciocchi, M.; Fantuzzi, N.; Reddy, J.N. Multiscale Approach for Three-Phase CNT/Polymer/Fiber Laminated Nanocomposite Structures. Polym. Compos. 2019, 40, 102-126. [CrossRef]

31. Fantuzzi, N. New insights into the strong formulation finite element method for solving elastostatic and elastodynamic problems. Curved Layer. Struct. 2014, 1, 93-126.

32. Civalek, O. Free vibration of carbon nanotubes reinforced (CNTR) and functionally graded shells and plates based on FSDT via discrete singular convolution method. Compos. Part B Eng. 2017, 111, 45-59. [CrossRef]

33. Civalek, O. Nonlinear dynamic response of laminated plates resting on nonlinear elastic foundations by the discrete singular convolution-differential quadrature coupled approaches. Compos. Part B Eng. 2013, 50, 171-179. [CrossRef]

34. Civalek, O.; Demir, C. Buckling and bending analyses of cantilever carbon nanotubes using the Euler-Bernoulli beam theory based on non-local continuum model. Asian J. Civ. Eng. 2011, 12, 651-661.

35. Civalek, O. Finite Element Analysis of Plates and Shells; Firat University: Elazı̆̆, Turkey, 1998. (In Turkish)

36. Uzun, B.; Numanoğlu, H.M.; Civalek, O. Free Vibration Analysis of BNNT with Different Cross-Sections via Nonlocal FEM. J. Comput. Appl. Mech. 2018, 49, 252-260.

37. Omurtag, M.H. Çubuk Sonlu Elemanlar; Birsen Yayinevi: Istanbul, Turkey, 2010. (In Turkish)

38. Işık, Ç. Mikro ve Nano Ölçekli Mekanik Sistemlerin Modellenmesinde Yerel Olmayan Sonlu Eleman Formülasyonu; Akdeniz Üniversitesi: Antalya, Turkey, 2018. (In Turkish)

(C) 2019 by the authors. Licensee MDPI, Basel, Switzerland. This article is an open access article distributed under the terms and conditions of the Creative Commons Attribution (CC BY) license (http://creativecommons.org/licenses/by/4.0/). 